\title{
Wirtschaftsdeutsch in China
}

\section{Eine Umfrage unter möglichen Arbeitgebern für Germanistikabsolventen}

\section{Jin Zhao}

\section{Einleitung}

Die schnelle Wirtschaftsentwicklung in China sowie die wirtschaftliche Globalisierung der ganzen Welt haben eine starke Zunahme von Niederlassungen deutscher Firmen in den großen chinesischen Städten zur Folge. Dies bietet den chinesischen DeutschlernerInnen neue Arbeitschancen. Besonders stark verändert hat sich das Berufsbild für chinesische GermanistInnen (vgl. Zhao 1996: 5). Nicht mehr nur als DolmetscherInnen und ÜbersetzerInnen oder HochschullehrerInnen sind sie tätig, sondern die meisten ergreifen Berufe in Unternehmen mit deutscher Kapitalbeteiligung als SekretärInnen, SachbearbeiterInnen, ChefassistentInnen usw. Sie sind nun mit neuen Berufsaufgaben in der Wirtschaft konfrontiert, die den Rahmen der traditionellen Germanistikausbildung mit ihrem Schwerpunkt auf Literatur, Linguistik und Landeskunde sprengen.

Um sich ausreichend für den zukünftigen Arbeitsmarkt zu qualifizieren und eine relativ günstige Ausgangslage für die berufliche Konkurrenz zu schaffen, besuchen viele GermanistikstudentInnen auf eigene Initiative hin Abendkurse im Bereich Wirtschaft. Auf der pädagogischen Ebene ist allmählich klar geworden, daß die germanistische Ausbildung zum Teil reformiert werden muß, wenn sie auf den heutigen Berufsmarkt abgestimmt sein soll.

Deswegen wird unter chinesischen GermanistInnen stark diskutiert, ob und in welcher Form das Germanistikstudium Wirtschaftsdeutsch-Elemente enthalten sollte. Einzelne Universitäten bzw. Institute haben schon aktiv darauf reagiert. Als eine der Pilotuniversitäten hat die Fremdsprachenuniversität Beijing beispielsweise wirtschaftsbezogene Lernbereiche in das Hauptstudium der Germanistik integriert. Die Deutschabteilung der Universität Wuhan hat auch einige Bereiche des Wirtschaftsdeutschen probeweise unterrichtet. An der Tongji-Universität wird seit der Gründung des Projekts »Wirtschaftsdeutsch « (im Jahr 1995) in Zusammenarbeit mit der PhilippsUniversität Marburg regelmäßig Wirtschaftsdeutsch im Blockseminar von deutschen Lektoren durchgeführt. Besonders zu beachten sind die Seminare zum Thema Wirtschaftsdeutsch unter der Leitung des Goethe-Instituts Zweigstelle Beijing, an denen über 10 Universitäten bzw. Institute teilnehmen und über bestimmte Faktoren im Unterricht des Wirtschaftsdeutschen wie Zielgruppen, The- 
men, Kontrolle usw. Meinungen austauschen, was die Unterrichtspraxis des Wirtschaftsdeutschen stark gefördert hat. Trotz all dieser individuellen und institutionellen Aktivitäten bleibt aber noch eine wichtige Frage unbeantwortet, nämlich was die Wirtschaft fordert. Mit anderen Worten: Es besteht die Gefahr, daß solche Bemühungen ohne die Beteiligung der Unternehmen ins Leere laufen. Um diesem Mangel abzuhelfen, habe ich im Rahmen des oben erwähnten Projekts zwischen Tongji und Marburg mit der Unterstützung von Frau Prof. Dr. Ruth Albert einen Fragebogen entworfen und im Herbst 1997 in der Wirtschaftsstadt Schanghai Erhebungen durchgeführt. Für Teile des Fragebogens haben wir eine wissenschaftlich abgesicherte Befragung aus Finnland zum Vorbild genommen (vgl. Bibliographie).

Ziel der vorliegenden Studie war es, die jetzige Arbeitssituation der chinesischen Germanistikabsolventen in der Wirtschaft sowie den Stellenwert der deutschen Sprache in den Unternehmen zu skizzieren und die Anforderungen seitens der Wirtschaft in bezug auf die sprachlichen Fertigkeiten wie auch die wirtschaftlichen Fachkenntnisse herauszufinden. Ich hoffe, daß die Ergebnisse dieser Umfrage eine der Voraussetzungen für die Erstellung eines Curriculums für den Unterricht in Wirtschaftsdeutsch in China schaffen können und den DeutschlehrerInnen bei der Planung des Unterrichts für Wirtschaftsdeutsch sowie zur Entwicklung von chinaspezifischen Lehrmaterialien von Nutzen sein werden.

\section{Die empirische Untersuchung}

Die empirische Untersuchung gliedert sich in zwei Teile: eine Fragebogenumfrage und ein halbstandardisiertes Interview mit ausgewählten Wirtschaftsver- tretern. Während sich der erste Teil im wesentlichen aus geschlossenen Fragen vom Typ Identifikation, Selektion und JaNein zusammensetzt und eine quantitative Untersuchung darstellt, sammelt der zweite konkrete Meinungen und gilt als qualitative Forschung, die die Interpretation des Zahlenmaterials unterstützt.

Die Untersuchungsgruppe wurde aus einer Unternehmensliste ausgewählt, die vom deutschen Generalkonsulat in Schanghai zusammengestellt wurde. Die Absicht der Auswahl war, die zu Befragenden möglichst prozentuell gleichgewichtig auf verschiedene Wirtschaftssektoren zu verteilen, damit diese Stichprobe eine Aussagekraft über ein breites Spektrum von Wirtschaftszweigen bekommt. In den Fragen 1-6 des Fragebogens geht es um Hintergrundinformationen über die Befragten sowie deren Firmen, während es in den Fragen 7-14 und 16 um die mündliche bzw. schriftliche Sprachverwendung und die Anforderungen an die wirtschaftlichen Fachkenntnisse geht. In den anderen Fragen werden die Ansichten über die Schwerpunktsetzung des benötigten Wirtschaftsdeutsch-Unterrichts bzw. der benötigten Kenntnisse erfragt.

Bei den 7 Interviews wurden die Vertretungen von großen, mittelständischen und kleinen Firmen berücksichtigt, und es kamen sowohl deutsche Geschäftsführer als auch chinesische MitarbeiterInnen mit Sprachstudienabschluß zu Wort. Im Interview-Leitfaden wurde eine inhaltliche Orientierung am Fragebogen vorgenommen, aber dabei wurde immer versucht, »Warum«-Fragen zu stellen, um die hinter den auf dem Fragebogen angegebenen Zahlen versteckten Stellungnahmen und Begründungen zu erfahren. Die Ergebnisse dieser Erhebung werde ich im folgenden bei der Darstellung der quantitativen Analyse mit berücksichtigen. 


\section{Ergebnisse}

\subsection{Rücklauf sowie Hintergrundinfor- mationen über die Befragten und deren Firmen}

Insgesamt 70 Fragebögen wurden in Schanghai verschickt. Der Rücklauf betrug 35 Fragebögen, davon kamen 18 aus der Industrie, 7 aus dem Handel, 6 aus dem Dienstleistungsbereich, 3 aus dem Bankwesen, 2 aus den sonstigen Berei- chen und 1 aus dem Transportwesen, wobei 2 Unternehmen jeweils 2 Wirtschaftssektoren abdekken (Frage 4a). Die Unternehmen, die den Fragebogen zurückgeschickt haben, verteilen sich gleichmäßig auf verschiedene Unternehmensformen, und zwar sind unter ihnen 7 Equity Joint Ventures, 7 Contractual Joint Ventures, 7 Wholly Foreign-Owned Enterprises und 14 Representative Offices (Frage $4 b$ ).

Tabelle zu Fragen 4b/5a/6: Größe der Unternehmen im Zusammenhang mit den Unternehmensformen und Umsatz mit Deutschland (in Prozent)

\begin{tabular}{||l|c|c|c|c|c|c|c||}
\hline & \multicolumn{5}{|c|}{ Unternehmensform } & \multicolumn{2}{c||}{$\begin{array}{c}\text { Geschäftsbeziehungen } \\
\text { mit Deutschland }\end{array}$} \\
\hline Größe & $\begin{array}{c}\text { Anteile un- } \\
\text { ter den be- } \\
\text { fragten } \\
\text { Unterneh- } \\
\text { men }\end{array}$ & $\begin{array}{c}\text { Equity } \\
\text { Joint } \\
\text { Venture }\end{array}$ & $\begin{array}{c}\text { Contrac- } \\
\text { tual Joint } \\
\text { Venture }\end{array}$ & $\begin{array}{c}\text { Wholly } \\
\text { Foreign } \\
\text { Owned } \\
\text { Enterprise }\end{array}$ & $\begin{array}{c}\text { Represen- } \\
\text { tative } \\
\text { Offices }\end{array}$ & $\begin{array}{c}\text { Umsatz } \\
\text { unter 50\% }\end{array}$ & $\begin{array}{c}\text { Umsatz } \\
\text { über } 50 \%\end{array}$ \\
\hline $\begin{array}{l}\text { kleine und } \\
\text { mittelstän- } \\
\text { dische Un- } \\
\text { ternehmen }\end{array}$ & 55,88 & 5,34 & 5,34 & 26,16 & 63,16 & 40 & 60 \\
\hline $\begin{array}{l}\text { große Un- } \\
\text { ternehmen }\end{array}$ & 44,14 & 42,68 & 42,86 & 7,14 & 7,14 & 76,92 & 23,08 \\
\hline
\end{tabular}

Wenn wir die Anzahl der MitarbeiterInnen als den entscheidenden Faktor bei der Größe der Unternehmen in Betracht ziehen und die Unternehmen mit weniger als 50 Angestellten als kleine bzw. mittelständische Unternehmen bezeichnen ${ }^{1}$, sind $55,88 \%$ von den befragten Unternehmen als klein bzw. mittelständisch charakterisiert. Werden die beiden Unternehmensgrößen im einzelnen betrachtet, fällt auf, daß die kleinen bzw. mittelständischen Unternehmen vorwiegend die
Form von Representative Office $(63,16 \%)$ und Wholly Foreign-Owned Enterprises $(26,16 \%)$ haben, während die großen Firmen in erster Linie Equity Joint Ventures $(42,6 \%)$ und Contractual Joint Ventures $(42,86 \%)$ darstellen (Frage 4b/5.a).

In bezug auf den Anteil am Umsatz an den Geschäftsbeziehungen mit Deutschland folgt überdies aus dieser Tabelle, daß $60 \%$ der kleinen bzw. mittelständischen Unternehmen das Geschäft vor allem zwischen Deutschland und China

1 In der Betriebswirtschaftslehre wird die Unternehmensgröße in Abhängigkeit von mindestens zwei der Abgrenzungsmerkmale (Bilanzsumme, Umsatzerlöse und Arbeitnehmer) entschieden. In bezug auf die Anzahl der Arbeitnehmer hat eine kleine Firma weniger als 50 und eine mittelgroße weniger als 250 MitarbeiterInnen (vgl. Bea et al. 1997: 436f.). Leider ist es schwer, dieses Kriterium in unserem Fall völlig zu übernehmen. Denn die Niederlassungen haben normalerweise weniger MitarbeiterInnen als die Mutterfirma. Ich bezeichne deshalb die Unternehmen mit weniger als 50 MitarbeiterInnen in China als klein bzw. mittelständisch. 
pflegen. Die meisten großen Firmen $(76,92 \%)$ erzielen demgegenüber durch die selbständige Geschäftsführung auf dem chinesischen sowie außerchinesischen Markt über die Hälfte des Umsatzes (Fragen $4 b / 6$ ).

Bemerkenswert ist die relativ hohe Zahl der fehlenden Angaben zu chinesischen MitarbeiterInnen in Frage 5.a. Das könnte einerseits darauf zurückgeführt werden, daß die Befragten die Frage nicht genau verstanden und somit nicht richtig beantworten konnten. Wahrscheinlich ist aber, daß die genaue Anzahl wegen der hohen Anzahl der Chinesen in der Firma nicht exakt gefaßt werden konnte. Aber aus den 54,29\% Fragebögen mit einer Antwort zu dieser Frage läßt sich trotzdem ablesen, daß chinesische MitarbeiterInnen meist über $50 \%$ der Arbeitskräfte ausmachen.

Da die Umfragebögen zu 55,88\% von den Geschäftsführenden und zu 25,47\% von den (Haupt)abteilungsleiterInnen ausgefüllt wurden (Frage 2), sind die durch die gekennzeichneten Zahlen präsentierten Einstellungen repräsentativ für das jeweilige Unternehmen. Aber sie scheinen Probleme zu haben in bezug auf Frage $5 \mathrm{~b}$. Denn in vielen Fällen wurde dieselbe Zahl bei "Fachpersonal mit Deutschkenntnissen « und bei »Germanisten « angegeben, oder sie ist einfach ausgelassen worden. Es scheint, als ob die Befragten in den meisten Fällen die Ausbildung der chinesischen MitarbeiterInnen nicht genau kennen würden. Das kann natürlich zum einen in der Konkretisierung der Fragestellung begründet sein, oder aber den Grund haben, daß manche GermanistInnen auch Facharbeiten übernommen haben. Das ist in den darauf folgenden Interviews zum Teil bestätigt worden:

Bemerkenswert ist es soweit, daß die Mitarbeiterin, die jetzt in Business Center arbeitet, sich wirklich hochgearbeitet hat. Es kann sein, daß Fachkenntnisse in ihrem
Studium abgedeckt waren. Aber ich vermute, daß es (eine) rein sprachliche Ausbildung war. (Deutscher Geschäftsführer eines großen Unternehmens aus der Dienstleistung)

Manche Germanisten arbeiten als Kaufleute bei uns in der Handelsabteilung, ich weiß nicht, ob sie als Fachpersonal oder Germanisten bezeichnet werden. (Chinesische Abteilungsleiterin eines großen Unternehmens aus dem Handel)

Trotz 6 unvollständiger und unlogischer Antworten wird die Prozentzahl in Frage 5.b wie folgt zusammengerechnet: Unter den chinesischen MitarbeiterInnen sind 73,84\% Fachpersonal ohne Deutschkenntnisse, 15,54\% Fachpersonal mit Deutschkenntnissen und 10,62\% Germanisten. Die hohe Prozentzahl des Fachpersonals ohne Deutschkenntnisse kann daraus resultieren, daß viele Angestellte Englisch sprechen und viele ArbeiterInnen in den großen Unternehmen gar keine Fremdsprachenkenntnisse besitzen und benötigen.

Für die möglichen Arbeitspositionen der chinesischen GermanistInnen wurden den Befragten in Frage 5.c drei Hauptabteilungen in den Unternehmen zum Ankreuzen vorgegeben. Die Ergebnisse haben gezeigt, daß mit $65,52 \%$ die meisten GermanistInnen ihre Berufe in der Verwaltungsabteilung ausüben und $24,15 \%$ von ihnen in der Handelsabteilung sowie 10,34\% in der Produktionsabteilung tätig sind. Diese überragenden Arbeitsmöglichkeiten für GermanistInnen in der Verwaltungsabteilung wurden ebenfalls in den Interviews hervorgehoben:

Die Germanisten werden in der Regel vorzüglich eingesetzt im Bereich vom Fremdsprachensekretariat oder von (der) Verwaltung, weil man zu (einer) klassischen Sekretärfunktion diese sprachliche und kulturelle Kompetenz mit einbezieht, welche im Deutschstudium sehr stark gegeben ist. (Deutscher Repräsentant einer kleinen Firma aus der Industrie) 
Am Anfang habe ich HR (human resource) gemacht in der Personalabteilung. ... Inzwischen hat mich unsere Firma zu einem chinesisch-europäischen Blockseminar über Management geschickt. ... Und jetzt bin ich Leiterin der Absatzabteilung. (Chinesische Abteilungsleiterin eines großen Joint Ventures aus dem Handel, ins Deutsche übersetzt)

Abschließend soll noch eine Bemerkung zu den Befragten gemacht werden: Zwar ist die Muttersprache der Befragten je- weils Chinesisch $(45,45 \%)$, Deutsch $(48,48 \%)$, Englisch $(3,04 \%)$ und Französisch $(3,03 \%)$, und die Fragebögen wurden in einer deutschen und einer chinesischen Version angeboten, 71,82\% der Fragebögen wurden trotzdem auf deutsch ausgefüllt. Es scheint so, daß die meisten Führungskräfte oder auch die SekretärInnen sowie SachbearbeiterInnen doch Deutsch können (Frage 2).

\subsection{Sprachverwendung in bezug auf die mündliche Kommunikation}

Tabelle zu Frage 7a: Verkehrssprache in der mündlichen Kommunikation (in Prozent)

\begin{tabular}{|l|c|c|c|c|c|}
\hline Sprache & 4 & 3 & 2 & 1 & ohne Angabe \\
\hline Deutsch & 20 & 14,29 & 42,87 & 11,42 & 11,42 \\
\hline Englisch & 54,28 & 22,86 & 22,86 & 0 & 0 \\
\hline Chinesisch & 5,71 & 8,57 & 20 & 45,72 & 20 \\
\hline
\end{tabular}

$4=$ fast immer, $3=$ recht viel, $2=$ etwas, $1=$ nie

Diese Tabelle vermittelt uns einen Überblick über die mündliche Kommunikation in den befragten Unternehmen. $\mathrm{Zu}$ erwähnen ist, daß die Antworten »ohne Angaben « mit »1 = nie« gleichgesetzt werden können, da in den Fragebögen oft nur die Kommunikationssprachen markiert und die Möglichkeit des Ankreuzens in der Spalte »1 « häufig außer acht gelassen wurden. Daraus läßt sich dann ersehen, daß in gut der Hälfte der Unternehmen Englisch als »fast immer" gesprochene Sprache angesehen wird. Die deutsche Sprache, die in 20\% der Unternehmen als Verkehrssprache verwendet wird, scheint jedoch mit $42,87 \%$ in recht vielen Unternehmen nur eine Ergänzungssprache zu sein. Werden die Unternehmen, wo Deutsch »fast immer« bzw. »recht viel« gesprochen wird, mit Frage 5.a in Korrelation gestellt, ergibt sich, daß $66,67 \%$ davon kleine bzw. mittelständische Firmen darstellen. Chinesischsprachige Unternehmen bleiben mit 5,71\% in der Minderheit. Diese sind vorwiegend kleine bzw. mittelständische Firmen im Bereich der Industrie und haben oft einen Chinesen als chief representative. Eine Germanistin hat in dem Interview ihre Firma so beschrieben:

Unser Manager ist eine Chinesin, die in Deutschland studiert hat und wieder zurückgekommen ist. Sie kontaktiert die Mutterfirma in Deutschland, natürlich auf Deutsch. Aber in unserem Büro sprechen wir nur Chinesisch. (Chinesische Mitarbeiterin einer mittelständischen Firma aus der Industrie, ins Deutsche übersetzt)

Eine interessante Frage ist nun weiterhin, in welchen Situationen die drei Sprachen in erster Linie gebraucht werden. Es wurden sechs Antwortoptionen in Frage 8 vorgegeben und die Befragten kreuzten jeweils eine der drei Sprachen an.

In den Antworten auf Frage 8 ist die Dominanz der englischen Sprache entsprechend ausgeprägt. Wenn wir aber unseren Blick auf die deutsche Sprache beschränken, ergibt sich daraus, daß sie am häufigsten in den informellen Zusam- 
Tabelle zu Frage 8: Situationsgebundene Verkehrssprache in der mündlichen Kommunikation (in Prozent)

\begin{tabular}{|l|c|c|c||}
\hline \hline Situationen & Deutsch & Englisch & Chinesisch \\
\hline Verhandlungen & 24,33 & 62,16 & 13,51 \\
\hline Besprechungen & 26,83 & 56,10 & 17,07 \\
\hline Telefonate & 29,27 & 56,10 & 14,63 \\
\hline Unternehmenspräsentationen & 15,62 & 71,88 & 12,50 \\
\hline Produktpräsentationen & 10,72 & 78,57 & 10,71 \\
\hline Informelle Zusammenkünfte & 40,91 & 43,18 & 15,91 \\
\hline
\end{tabular}

menkünften verwendet wird. Selbst in den deutschen Unternehmen, in denen Englisch als Geschäftssprache dient, sprechen die deutschen MitarbeiterInnen gern in ihrer Muttersprache mit ihren chinesischen KollegInnen. Weitere Informationen aus der Wirtschaft besagen:

Da wir Kontakt mit anderen Filialen in Australien, Frankreich usw. haben, muß unsere Arbeitssprache Englisch sein. Aber wenn man Deutsch noch zusätzlich kann, kann man sich besser mit dem deutschen Chef verstehen. (Chinesische Abteilungsleiterin eines großen Joint Venture aus dem Handel, ins Deutsche übersetzt)

Unsere Verkehrssprache ist Englisch. ... Natürlich ist es für die deutschen Delegierten angenehmer, in ihrer Muttersprache zu kommunizieren, jedoch dürfte man selten auf chinesische MitarbeiterInnen treffen, die einen in Deutsch diktierten Brief auch ins Englisch übersetzen könnten. (Deutsche Mitarbeiterin einer großen Firma aus dem Handel, aus dem Begleitbrief zu einem Fragebogen)

Bei Telefonaten, Besprechungen und Verhandlungen darf die deutsche Sprache nicht vernachlässigt werden. Aber genau auf diesen Gebieten müssen die GermanistInnen ihre mündliche Fertigkeit nach den Interviews noch optimieren. Die Angaben zu Frage 7.b, daß 30,30\% der chinesischen MitarbeiterInnen in der mündlichen Kommunikation auf Deutsch Schwierigkeiten haben und $15,15 \%$ da- von sogar große Schwierigkeiten haben, beziehen sich vor allem auf diese Gebiete:

Telefonate sind sicher ein sehr großes Problem. ... Telefonieren ist eine Ubung, die auch geschult werden sollte, auch muß.... In Deutschland gibt es z. B. für Fremdsprachsekretärin(nen) Kurse, über das richtige Telefonieren, das richtige Eingehen auf den Gesprächspartner über das Medium Telefon geübt wird. ... Wenn hier die chinesischen Mitarbeiter deutsche Firmen, deutsche Techniker, die bei den deutschen Firmen hier ja arbeiten, kontaktieren, da gibt es manchmal schon Verständigungsschwierigkeiten. (Deutscher Repräsentant einer kleinen Firma aus der Industrie)

Ihnen (den chinesischen Mitarbeitern) fehlt die Interaktionsfähigkeit im Gespräch. Es ist die Sache der praktischen Übungen. (Deutscher Repräsentant einer kleinen Firma aus der Industrie)

In den Fällen der Unternehmens- und Produktpräsentationen spielt die deutsche Sprache keine wichtige Rolle, wobei Englisch mit jeweils 71,88\% und 78,57\% unstreitig als vorrangige Präsentationssprache benutzt wird.

Gedolmetscht wird Deutsch (48\%) fast genau so viel wie Englisch (52\%). Die Gewichtung von Dolmetschen ist aber recht heterogen: Während in der Hälfte der Unternehmen dem Dolmetschen eine sehr große $(25,7 \%)$ bis große $(25,7 \%)$ Bedeutung zugeteilt wird, schätzten die übrigen Unternehmen diese Aufgabe mittelgroß $(20 \%)$ sowie gering $(28,57 \%)$ ein (Frage 9$)$. 


\subsection{Sprachverwendung in bezug auf die schriftliche Kommunikation}

Tabelle zu Frage 10: Verkehrssprache in der schriftlichen Kommunikation (in Prozent)

\begin{tabular}{|l|c|c|c|c|c||}
\hline Sprachen & 4 & 3 & 2 & 1 & ohne Angabe \\
\hline Deutsch & 14,29 & 17,14 & 34,29 & 22,26 & 11,42 \\
\hline Englisch & 54,29 & 25,71 & 14,29 & 5,71 & 0 \\
\hline Chinesisch & 5,71 & 11,432 & 22,86 & 40 & 20 \\
\hline
\end{tabular}

$4=$ fast immer, $3=$ recht viel, $2=$ etwas, $1=$ nie

Bei den Antworten auf Frage 10, in der es um die schriftliche Verwendungshäufigkeit der drei Sprachen Deutsch, Englisch und Chinesisch in den befragten Unternehmen geht, weisen die Meinungen im Vergleich mit denen zur mündlichen Kommunikation (siehe Tabelle 7.a) keine größeren Unterschiede auf. Zwar wird bei der schriftlichen Kommunikation Deutsch weniger »fast immer« $(14,29 \%)$ verwendet als bei der mündlichen $(20 \%)$, aber durch die Zunahme der deutschen Sprache in der Spalte $» 3=$ recht viel $«$ $(17,14 \%)$ wird Deutsch fast genau so viel schriftlich wie mündlich benutzt.

Von Interesse erscheint mir ebenfalls die Frage, ob sich die Unternehmens- größe auf die schriftliche Kommunikationssprache ähnlich wie auf die mündliche auswirkt. Die Auswertung zeigt, daß die Unternehmen, in denen Deutsch in der schriftlichen Kommunikation »fast immer « und »recht viel« gebraucht wird, zu $72,73 \%$ als klein bzw. mittelständisch bezeichnet werden können.

Mit der Frage 11 sollte herausgefunden werden, welche Textsorten in der deutschen Sprache am häufigsten geschrieben bzw. gelesen werden. Dabei wurden zehn Textsorten zur Quantifizierung vorgegeben. Im folgenden richten wir unseren Blick zuerst auf die geschriebenen Texte.

Tabelle zu Frage 11 A: Geschriebene Texte im Geschäftsleben - Bedarf (in Prozent)

\begin{tabular}{|l|r|r|r|r||}
\hline Textsorte & \multicolumn{1}{|c|}{4} & \multicolumn{1}{|c||}{2} & 1 \\
\hline a. Geschäftsbereich & 29,41 & 5,88 & 14,71 & 50 \\
\hline $\begin{array}{l}\text { b. Sonstige Briefe (Einladungen, } \\
\text { Danksagungen u. ä.) }\end{array}$ & 23,53 & 20,59 & 8,82 & 47,06 \\
\hline c. Berichte verschiedener Art & 20,59 & 11,76 & 17,65 & 50 \\
\hline d. Protokolle, Memoranden & 14,71 & 11,76 & 8,82 & 64,71 \\
\hline e. Anweisungstexte & 14,71 & 11,76 & 14,71 & 58,82 \\
\hline f. Verträge & 5,88 & 11,76 & 8,82 & 73,54 \\
\hline g. Produkt- und Betriebsbroschüren & 8,82 & 14,71 & 11,76 & 64,71 \\
\hline h. Pressemitteilungen & 5,88 & 11,76 & 14,71 & 67,65 \\
\hline i. Journalistische Wirtschaftstexte & 5,88 & 5,88 & 5,88 & 82,35 \\
\hline j. Artikel in Fachzeitschriften & 5,88 & 2,94 & 8,82 & 82,35 \\
\hline
\end{tabular}

4 = wöchentlich, 3 = monatlich, 2 = jährlich, $1=$ nie 
Da in mehr als der Hälfte der Unternehmen $(54,2 \%)$ »fast immer « schriftlich über Englisch kommuniziert wird (Frage 10), hat diese Fragestellung für sie keine Bedeutung mehr. Kein Wunder, daß sich der prozentuale Anteil unter »nie« dementsprechend meistens auf mehr als 50\% beläuft.

Was mich interessiert, sind aber die relativen Beziehungen zwischen den Textsorten. Mit 44,12\% sind die "Sonstigen Briefe « die am häufigsten geschriebene Textsorte (wöchentlich + monatlich), gefolgt von den Geschäftsbriefen und Berichten. Protokolle/Memoranden und Anweisungstexte rangieren mit gleichen Prozentzahlen an vierter Stelle. Für andere Textsorten gibt es nur wenige Nennungen. Der Schreibprozeß verläuft wie folgt: 45,83\% der Befragten haben erklärt, daß Texte am häufigsten von den chinesischen MitarbeiterInnen von Anfang bis Ende geschrieben werden. Auf Deutsch entwerfen und dann überarbeiten lassen ist die am zweithäufigsten genannte Variante $(37,5 \%)$, und Mustertexte werden nur von 17,39\% der Befragten für den am häufigsten benutzten Schreibablauf gehalten. Nicht üblich hingegen ist, daß ein Text oder Entwurf auf Chinesisch geschrieben und danach ins Deutsche übersetzt wird (Frage 12).

Das häufig selbständige Schreiben der Texte von den chinesischen MitarbeiterInnen bedeutet jedoch nicht, daß deren Deutsch einwandfrei ist. Insgesamt 77,78\% der Befragten haben darauf hingewiesen, daß die chinesischen MitarbeiterInnen das Schreiben deutschsprachiger Texte als schwierig empfinden (Frage 13.a). Die Schwierigkeiten liegen hier wesentlich im Stil $(35,71 \%)$, wobei die Probleme von Grammatik $(23,81 \%)$, Wortschatz $(21,43 \%)$ und Fachlexik $(19,05 \%)$ auch nicht selten auftreten (Frage 13.b). Die Frage 13.c ließen die meisten unbeantwortet. 9 Befragte haben Kenntnisse der chinesischen MitarbeiterInnen über die Unterschiede von Briefen, Telefax und e-mail bejaht, während sich die anderen 5 gegenteilig geäußert haben. Die Ursachen könnten darin liegen, daß die Frage nach den konkreten Tätigkeiten der chinesischen MitarbeiterInnen für befragte Chefs nicht zu beantworten war, weil sie mehrere Stufen höher in der Firmenhierarchie angesiedelt waren.

In Frage 15 wurden die Vertreter aus der Wirtschaft danach gefragt, für wie wichtig das Behandeln der oben aufgelisteten Textsorten im Unterricht gehalten wird. Sie wurden gebeten, dies auf einer Skala von 5 bis $1 \mathrm{zu}$ bewerten. Die folgende Tabelle (s. S. 590) präsentiert die Meinungen zum Schreibunterricht.

In dieser Tabelle ist auffällig, daß die prozentuale Anzahl der Ansicht, die aufgelisteten Textsorten seien nicht zu behandeln, im Vergleich mit Tabelle $11 \mathrm{~A}$ in der Spalte 1 wesentlich reduziert ist, auch wenn Unbeantwortet-Lassen als NichtBehandeln gedeutet wird. Das könnte damit begründet werden, daß selbst die von der englischen Sprache geprägten deutschen Unternehmen den Schreibunterricht in Deutsch als wichtig beurteilen. In dem Interview mit einer Abteilungsleiterin von einem großen Unternehmen wurde meine Hypothese weiter bestätigt. Zwar sei Englisch bei ihr zweifelsohne die Geschäftssprache, aber wenn man mit dem deutschen Berater korrespondiere, könne es auch über Deutsch verlaufen. Leider könnten die meisten Mitarbeiter kein Deutsch.

Es ist weiterhin ersichtlich, daß das Schreiben von Geschäftsbriefen im Unterricht als das wichtigste eingeschätzt wird, was aber angesichts des großen Bedarfs (Tabelle 11 A) keine Überraschung bereitet.

Wenn wir den Bedarf an Texten, die wöchentlich und/bzw. monatlich geschrieben werden, mit den Wünschen zur Be- 
Tabelle zu Frage 15 A: Vorschläge für zu schreibende Texte im Unterricht - Wunsch (in Prozent)

\begin{tabular}{||l|c|c|c|c|c|c||}
\hline Textsorte & 5 & 4 & 3 & 2 & 1 & ohne Angaben \\
\hline a. Geschäftsbereich & 54,29 & 20 & 5,71 & 0 & 2,86 & 17,14 \\
\hline $\begin{array}{l}\text { b. Sonstige Briefe (Einladun- } \\
\text { gen, Danksagungen u. ä.) }\end{array}$ & 17,14 & 22,86 & 25,71 & 11,43 & 8,57 & 17,14 \\
\hline c. Berichte verschiedener Art & 17,14 & 25,71 & 20 & 14,29 & 2,86 & 17,14 \\
\hline d. Protokolle, Memoranden & 25,71 & 31,43 & 8,57 & 2,86 & 14,29 & 17,14 \\
\hline e. Anweisungstexte & 5,71 & 17,14 & 17,14 & 14,29 & 25,71 & 20 \\
\hline f. Verträge & 8,57 & 34,29 & 11,43 & 8,57 & 20 & 17,14 \\
\hline $\begin{array}{l}\text { g. Produkt- und Betriebsbro- } \\
\text { schüren }\end{array}$ & 8,57 & 14,29 & 11,43 & 17,14 & 28,57 & 20 \\
\hline h. Pressemitteilungen & 2,86 & 8,57 & 11,43 & 25,71 & 31,43 & 20 \\
\hline $\begin{array}{l}\text { i. Journalistische Wirtschafts- } \\
\text { texte }\end{array}$ & 2,86 & 8,57 & 17,14 & 17,14 & 34,29 & 20 \\
\hline j. Artikel in Fachzeitschriften & 2,86 & 5,71 & 22,86 & 22,86 & 25,71 & 20 \\
\hline
\end{tabular}

$5=$ sollte vorrangig im Unterricht behandelt werden

$4=$ ein beträchtlicher Teil des Unterrichts sollte sich damit befassen

3 = wenigstens ein kleiner Teil des Unterrichts sollte dafür verwendet werden

$2=$ wenn zeitlich möglich, könnten diese Texte etwas behandelt werden

$1=$ lohnt sich nicht, diese Texte im Unterricht zu behandeln

handlung im Unterricht (vorrangig + ein beträchtlicher Teil) vergleichen, ergibt sich ein differenziertes Bild (Die Buchstaben kennzeichnen die jeweiligen Textsorten wie in Tabellen $11 \mathrm{~A}$ und 15A):

Abbildung zu Frage 11 A/15 A: Vergleich von Bedarf und Wunsch in bezug auf Schreiben (in Prozent)

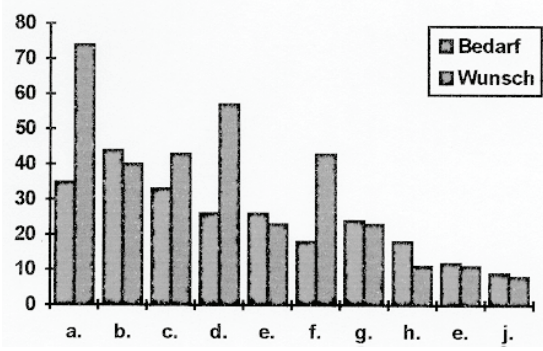

Der Wunsch, die Textsorten Geschäftsbriefe, Protokolle/Memoranden und Verträge im Schreibunterricht zu erlernen, scheint sehr hoch angesiedelt $\mathrm{zu}$ sein. Auch wird die Behandlung von Berichten verschiedener Art oft gewünscht. Auffällig sind hier die Beurteilung der Verträge: Obwohl diese seltener gebraucht werden, sollten sie trotzdem eine zentralere Rolle im Unterricht spielen. Aus Neugier habe ich bei den Interviews wiederum die Wichtigkeit des Schreibens von Verträgen beurteilen lassen, die Antworten sind jedoch alle negativ. Es wurde oft darauf hingewiesen, daß dem Verstehen sowie der Analyse der Bausteine von Verträgen hohe Bedeutung beigemessen wird, das Schreiben der Verträge wird jedoch oft als Aufgaben des Fachpersonals (z. B. Juristen) betrachtet. Möglicherweise geht man davon aus, daß die Übung im Schreiben von Verträgen die Analysefähigkeit bei vorliegenden Verträgen verbessert.

In bezug auf gelesene Texte gibt es noch zwei weitere Tabellen: 
Tabelle zu Frage 11 B: Gelesene Texte im Geschäftsleben - Bedarf (in Prozent)

\begin{tabular}{||l|c|c|c|c||}
\hline Textsorte & 4 & 3 & 2 & 1 \\
\hline a. Geschäftsbereich & 34,29 & 11,43 & 11,43 & 42,85 \\
\hline $\begin{array}{l}\text { b. Sonstige Briefe (Einladungen, } \\
\text { Danksagungen u. ä.) }\end{array}$ & 31,43 & 17,14 & 11,43 & 40 \\
\hline c. Berichte verschiedener Art & 20 & 20 & 14,29 & 45,71 \\
\hline d. Protokolle, Memoranden & 20 & 17,14 & 11,43 & 37,13 \\
\hline e. Anweisungstexte & 25,71 & 14,29 & 20 & 40 \\
\hline f. Verträge & 14,29 & 17,14 & 14,29 & 54,28 \\
\hline g. Produkt- und Betriebsbroschüren & 14,29 & 28,57 & 20 & 37,14 \\
\hline h. Pressemitteilungen & 17,14 & 28,57 & 8,57 & 45,71 \\
\hline i. Journalistische Wirtschaftstexte & 8,57 & 20 & 20 & 51,43 \\
\hline j. Artikel in Fachzeitschriften & 8,57 & 25,71 & 14,29 & 51,43 \\
\hline
\end{tabular}

4 = wöchentlich, 3 = monatlich, 2 = jährlich, $1=$ nie

Tabelle zu Frage 15 B: Vorschläge für zu lesende Texte im Unterricht - Wunsch (in Prozent)

\begin{tabular}{|l|c|c|c|c|c|c||}
\hline Textsorte & 5 & 4 & 3 & 2 & 1 & $\begin{array}{c}\text { ohne } \\
\text { Angaben }\end{array}$ \\
\hline a. Geschäftsbereich & 48,58 & 25,71 & 8,57 & 0 & 0 & 17,14 \\
\hline $\begin{array}{l}\text { b. Sonstige Briefe (Einladungen, } \\
\text { Danksagungen u. ä.) }\end{array}$ & 14,29 & 20 & 25,71 & 14,29 & 8,57 & 17,14 \\
\hline c. Berichte verschiedener Art & 17,14 & 37,14 & 14,29 & 11,43 & 5,71 & 17,14 \\
\hline d. Protokolle, Memoranden & 14,29 & 37,14 & 11,43 & 2,86 & 14,29 & 20 \\
\hline e. Anweisungstexte & 5,71 & 20 & 34,29 & 11,43 & 11,43 & 20 \\
\hline f. Verträge & 8,57 & 25,71 & 28,57 & 2,86 & 14,29 & 20 \\
\hline g. Produkt- und Betriebsbroschüren & 14,29 & 14,29 & 20 & 17,14 & 14,29 & 20 \\
\hline h. Pressemitteilungen & 5,71 & 8,57 & 28,57 & 17,14 & 20 & 20 \\
\hline i. Journalistische Wirtschaftstexte & 5,71 & 22,86 & 22,86 & 11,43 & 17,14 & 20 \\
\hline j. Artikel in Fachzeitschriften & 11,43 & 22,86 & 17,14 & 17,14 & 8,57 & 22,86 \\
\hline
\end{tabular}

$5=$ sollte vorrangig im Unterricht behandelt werden

$4=$ ein beträchtlicher Teil des Unterrichts sollte sich damit befassen

$3=$ wenigstens ein kleiner Teil des Unterrichts sollte dafür verwendet werden

2 = wenn zeitlich möglich, könnten diese Texte etwas behandelt werden

$1=$ lohnt sich nicht, diese Texte im Unterricht zu behandeln

Stellt man einen Vergleich zwischen Tabelle $11 \mathrm{~B}$ und Tabelle $11 \mathrm{~A}$ an, kommt man zum Ergebnis, daß einzelne Text- sorten im Geschäftsleben mehr gelesen als geschrieben werden. Bemerkenswert ist außerdem, daß unter den fünf am 
häufigsten gelesenen Textsorten (wöchentlich + monatlich) statt $»$ Berichte verschiedener Art « und »Protokolle/ Memoranden « beim Schreiben zwei andere Textsorten als relevant gelten, die Rangordnung sieht folgendermaßen aus: Sonstige Briefe, Geschäftsbriefe, Pressemitteilungen, Produkt-/Betriebsbroschüren und Anweisungstexte. Die Relevanz des Lesens von Pressemitteilungen sowie Produkt-/Betriebsbroschüren ist so $\mathrm{zu}$ verstehen, daß die MitarbeiterInnen sich dadurch einarbeiten und inner- bzw. interbetrieblich informieren könnten.

Abbildung zu Frage 11 B/15 B: Vergleich von Bedarf und Wunsch in bezug auf Lesen (in Prozent)

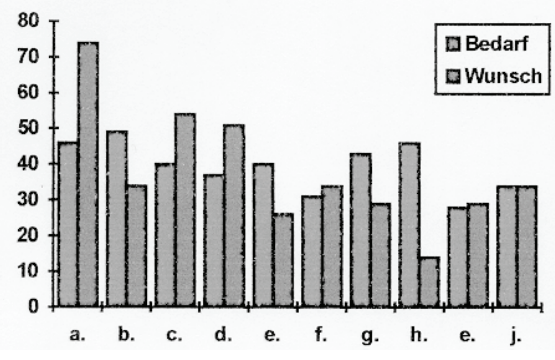

Die Korrelation von Bedarf im Beruf (wöchentlich + monatlich) und Wünschen an den Unterricht (vorrangig + ein beträchtlicher Teil) beim Lesen präsentiert ein ähnliches Bild wie beim Schreiben. Dem Leseverständnis von Geschäftsbriefen, Berichten verschiedener Art, Protokollen/ Memoranden sowie Verträgen soll nach Ansicht der Befragten weiterhin im Unterricht Aufmerksamkeit geschenkt werden, wobei das Lesen von sonstigen Briefen, Anweisungstexten, Produkt-/Betriebsbroschüren sowie Pressemitteilungen bei den chinesischen MitarbeiterInnen bereits jetzt zufriedenstellend zu sein scheint, oder es wird nur nicht so wichtig genommen.
Da journalistische Wirtschaftstexte und Artikel in Fachzeitschriften eher gelesen als geschrieben werden, werden auch das Training des Leseverständnisses dieser beiden Texttypen entsprechend gefordert.

Übersetzungsaufgaben fallen in der einen Hälfte der Unternehmen zwischen Englisch und Chinesisch, in der anderen Hälfte zwischen Deutsch und Chinesisch an, dabei ist deren Anteil in den jeweiligen Firmen von den 51,43\% der Befragten als »sehr groß übrigen als »mittelgroß « bis »gering « beurteilt worden.

\subsection{Wirtschaftliche Fachkenntnisse}

In Frage 16 wurden die Befragten um eine Meinung zu den notwendigen wirtschaftlichen Fachkenntnissen der chinesischen MitarbeiterInnen auf einer Rangskala von 5 (unverzichtbar) bis 1 (überflüssig) gebeten. Zu 9 bzw. 11 Teilbereichen aus der Betriebswirtschaftsund Volkswirtschaftslehre wurde die Relevanz für die Ausbildung der zukünftigen MitarbeiterInnen mit einem Germanistikabschluß erfragt. $\mathrm{Zu}$ erwähnen ist, daß die Einschätzung von den konkreten Bedürfnissen des jeweiligen Unternehmens oder der dem Befragten angehörigen Abteilung beeinflußt sein kann. Denn der Bedarf an den wirtschaftlichen Teilkenntnissen gilt oft als branchenbzw. positionsabhängig. Trotzdem werden die Antworten auf diese Frage immerhin für richtungsweisend gehalten.

20\% der Befragten ließen Frage 16 unbeantwortet, und zwar mit Bemerkungen, daß dort nur Ingenieure arbeiteten oder es innerbetrieblich unterschiedliche Belange gebe. Unter Betriebswirtschaftslehre werden folgende Bereiche als am wichtigsten eingestuft (unverzichtbar + sehr wichtig): Grundbegriffe (betriebliche Produktionsfaktoren, Funktionsbereiche wie Produktion, Absatzwirtschaft, Inve- 
Tabelle zu Frage 16 a: Betriebswirtschaftslehre (in Prozent)

\begin{tabular}{|l|c|c|c|c|c|c||}
\hline Betriebswirtschaftslehre & 5 & 4 & 3 & 2 & 1 & ohne Angabe \\
\hline a. Grundbegriffe & 31,43 & 22,86 & 11,43 & 8,57 & 5,71 & 20 \\
\hline $\begin{array}{l}\text { b. Industriebetriebs- } \\
\text { lehre }\end{array}$ & 17,14 & 22,86 & 17,14 & 11,43 & 11,43 & 20 \\
\hline c. Handelsbetriebslehre & 14,29 & 28,57 & 25,71 & 8,57 & 2,86 & 20 \\
\hline d. Bankbetriebslehre & 5,71 & 5,71 & 20 & 34,29 & 14,29 & 20 \\
\hline e. Personalwirtschaft & 5,71 & 8,57 & 17,14 & 34,29 & 14,29 & 20 \\
\hline f. Finanzbuchhaltung & 2,86 & 25,71 & 22,86 & 22,86 & 5,71 & 20 \\
\hline $\begin{array}{l}\text { g. Kalkulation und } \\
\text { Kostenrechnung }\end{array}$ & 8,75 & 22,86 & 11,43 & 31,43 & 5,71 & 20 \\
\hline h. Transportwesen & 2,86 & 5,71 & 22,86 & 37,14 & 14,29 & 20 \\
\hline j. Betriebliche Steuer- & 2,86 & 17,14 & 8,57 & 34,29 & 14,29 & 20 \\
\hline
\end{tabular}

$5=$ unverzichtbar, $4=$ sehr wichtig, $3=$ wichtig, $2=$ weniger wichtig, $1=$ überflüssig

Tabelle zu Frage 16 B: Volkswirtschaftslehre (in Prozent)

\begin{tabular}{|l|r|r|r|r|c|c||}
\hline Volkswirtschaftslehre & \multicolumn{1}{|c|}{5} & 4 & 3 & 2 & 1 & ohne Angabe \\
\hline a. Grundbegriffe & 11,43 & 22,86 & 20 & 14,29 & 11,43 & 20 \\
\hline b. Wirtschaftskreislauf & 2,86 & 14,29 & 22,86 & 20 & 20 & 20 \\
\hline $\begin{array}{l}\text { c. Volkswirtschaftliche Gesamt- } \\
\text { rechnung }\end{array}$ & 5,71 & 14,29 & 8,57 & 31,43 & 20 & 20 \\
\hline d. Markt und Preis & 8,57 & 31,43 & 22,86 & 5,71 & 11,43 & 20 \\
\hline e. Geld, Kredit, Banksystem & 5,71 & 22,86 & 31,43 & 11,43 & 8,57 & 20 \\
\hline f. Außenwirtschaftslehre und & 5,71 & 8,57 & 31,43 & 14,29 & 20 & 20 \\
\hline \begin{tabular}{l} 
g. Kolitik \\
\hline h. Wirtschaftssystem
\end{tabular} & 5,71 & 8,57 & 22,86 & 28,57 & 14,29 & 20 \\
\hline i. Steuern in Deutschland und & 2,86 & 14,20 & 20 & 22,86 & 20 & 20 \\
\hline China & 2,86 & 11,43 & 28,57 & 24,71 & 11,43 & 20 \\
\hline j. Wirtschaftspolitik & 5,71 & 20 & 20 & 22,86 & 11,43 & 20 \\
\hline \begin{tabular}{l} 
k. Wirtschaftsentwicklung, -struk- \\
\hline
\end{tabular} & & 25,71 & 25,71 & 8,57 & 20 \\
\hline
\end{tabular}

stition und Finanzierung etc.), Handelsbetriebslehre (insbesondere BWL des Außenhandelsbetriebes), Finanzbuchhal- tung und Kalkulation und Kostenrechnung. Bezüglich der Volkswirtschaftslehre sind im folgenden die wichtigsten 
Faktoren genannt: Grundbegriffe (Bedürfnisse, Güter, Produktion, Konsum, Knappheit, Wirtschaftssubjekte etc.), Markt und Preis, Geld/Kredit/Banksystem, Wirtschaftssysteme und Wirtschaftsentwicklung/-struktur in Deutschland und China. Auffällig ist, daß im allgemeinen die Wichtigkeit von BWL erheblich höher gesehen wird als die von VWL.

Weiterhin wurde dieses Meinungsbild in den Interviews konkretisiert. Folgende Auszüge können diese Statistik präzisieren:

Es gibt zunächst die Grundbegriffe, die jeder auch versteht. Wie ist ein Unternehmen aufgebaut, was sind (die) Produktionsfaktoren, wie hängen Produktion, Investition, Finanzierung und Absatz zusammen, wie funktioniert eigentlich ein Unternehmen. Das ist für die Germanistikstudenten am wichtigsten. (Deutscher Geschäftsführer einer kleinen Firma aus der Dienstleistung)

Handelsbetriebslehre ist immer wichtig für eine Firma, die im Ausland arbeitet. (ebd.)

Bei VWL wird mir wichtig, daß jemand schon ein bißchen über (das) Zeitgeschehen informiert ist, daß jemand auch schon über Wirtschaftspolitik, Wirtschaftsentwicklung im Vergleich China-Deutschland Bescheid weiß. Grundbegriffe sind ja wichtig, als Basis (um) dieses Zeitgeschehen zu verstehen. (Deutscher Geschäftsführer einer kleinen Firma aus dem Handel)

Aber die Germanistikstudenten sollten sich lieber mit Managementfähigkeit, mit BWLKenntnissen auseinandersetzen. (Chinesische Abteilungsleiterin eines großen Unternehmens aus dem Handel)

Es gibt dann Probleme, sie (die Germanisten) ohne (eine) vorherige Ausbildung oder Schulung direkt in wirtschaftliche Tätigkeit einzubinden, weil in der Regel dieses Hintergrundwissens über die einfachen Zusammenhänge von BWL, wie z. B. Zusammensetzung von Kosten, Service fehlt. (Deutscher Geschäftsführer eines großen Unternehmens aus der Dienstleistung)
Außerdem haben die Interviewten ihre Meinungen darüber geäußert, in welchem Umfang diese Fachkenntnisse im Studium vermittelt werden sollen:

Ich würde also darauf achten, bei einer Vorstellung eines Mitarbeiters, ob er bereits Erfahrungen hat, wenn er keine Erfahrungen hat, ob er Möglichkeiten mitbringt, von seiner Ausbildung, daß man davon ausgehen kann, daß die Basis geschaffen ist, - für wirtschaftliche Grundzusammenhänge. Das halte ich für sehr wichtig. (Deutscher Repräsentant einer kleinen Firma aus der Dienstleistung)

Das kommt immer darauf an, für was man arbeitet. ... Je breiter man sich bildet, desto breiter ist später auch die Chance, einen Ausbildungsplatz zu bekommen. (Deutscher Geschäftsführer eines großen Unternehmens aus der Dienstleistung)

Es reicht nicht, nur die wirtschaftlichen Ausdrücke zu beherrschen. Aber ich nehme an, daß wenn jemand Wirtschaftsdeutsch lernt, dann lernt er gleichzeitig auch ein bißchen die Betriebswirtschaft, denn er muß wissen, was diese Wörter bedeuten, was dahinter steckt. (Deutscher Geschäftsführer einer kleinen Firma aus der Dienstleistung)

Es macht ja vielleicht nicht unbedingt Sinn, sich als Germanistikstudent zusätzlich noch auf Bankbetriebslehre oder Transportwesen zu spezialisieren. Sondern Germanistikstudenten sollten versuchen, eine doch relativ breite BWL-Zusatzbildung zu bekommen. Er wird bei dem Bewerbungsgespräch darauf hin getestet werden, und wenn er da zu verstehen gibt, daß er eigentlich im Grunde von Management eine Ahnung hat, dann wird jeder Chef sagen, das Spezielle können wir (ihm) auch beibringen. Wichtig ist, daß er erst einmal eine Grundlage hat (ebd.).

Man muß sich eigentlich volkswirtschaftlich aus den Medien bilden. Man muß das volkswirtschaftliche Geschehen also verfolgen. ... So ein richtiges tiefgehendes Volkswirtschaftsstudium wird sehr schnell theoretisch. Es wird nur noch 5\% von dem gebraucht, was man eigentlich gelernt hat. (Deutscher Repräsentant einer kleinen Firma aus der Industrie) 


\subsection{Die mangelnden Qualifikationen}

Die Antworten auf Frage 17 deuten darauf hin, was im Deutschunterricht an der Hochschule behandelt werden sollte, um die Deutschstudenten am besten für die kommenden Aufgaben im Geschäftsleben vorzubereiten. Unter den vier angegebenen Qualifikationen wurde »mündliche Kommunikation « $(32,56 \%)$ als das Wichtigste empfohlen. Mit weniger Abstand folgen »Wirtschaftliche Fachkenntnisse« (30,23\%), während »schriftliche Kommunikation « $(22,09 \%)$ und $»$ Kultur der deutschsprachigen Länder « $(15,12 \%)$ als weniger wichtig angesehen wurden. Ein ähnliches Meinungsbild schlägt sich in den Interviews nieder. Die Fähigkeit der mündlichen Kommunikation wurde wiederholt als Defizit der chinesischen Mitarbeiter erwähnt. Zwar könnten sie normalerweise schon grammatisch fehlerfrei sprechen, aber leider nicht situationsgebunden. Dadurch würden dann Mißverständnisse hervorgerufen. Demzufolge wurden praxisnahe Übungen im Unterricht explizit und dringend vorgeschlagen. Außerdem wurde als negativ empfunden, daß man trotz guter Sprachkenntnisse keine Ahnung über den Betriebsablauf habe. Gute Sprachkenntnisse seien zwar Kriterium, aber die Wirtschaft könne solche Leute nicht einstellen, die nur auf Deutsch »Guten Tag « sagen oder über das Wetter reden könnten. Nur wer gutes Deutsch spreche und zugleich Grundverständnis für Wirtschaft habe, könne die Marktlücke füllen.

Die interkulturelle Kompetenz, die im Deutschstudium schon sehr stark gegeben sei, wurde aber trotzdem als unverzichtbar betrachtet. Die deutschen Unternehmen brauchten Mitarbeiter, die Ausländer und die deutsche Kultur verstehen würden. Darüber hinaus sei das Wissen über interkulturelles Management und interkulturelle wirtschaftliche Landeskunde jedoch wichtiger.
Als Ergänzung wurden Handlungs- und selbständige Arbeitsfähigkeit sowohl auf den Fragebögen vermerkt als auch in den Interviews akzentuiert. Da die wirtschaftliche Tätigkeit rational und gewinnorientiert sei, müßten die Mitarbeiter ihre Kenntnisse auch in die Praxis umsetzen können. Es sei sehr schwierig, auch gut ausgebildete Absolventen einzuarbeiten, wenn sie sehr stark chinesische Arbeitsweisen gewohnt seien, die sehr wenig Wert auf Unabhängigkeit und selbständiges Handeln legten.

\section{Zusammenfassung und Ausblick}

Sichere Jobs in der Wirtschaft sind für die GermanistInnen Glücksfälle, insbesondere für die chinesischen GermanistInnen, denen Berufsfelder, die in Deutschland mit dem Germanistikstudium angestrebt werden (z.B. Lektor im Verlag, Angestellter im Rundfunk bzw. im Fernsehen oder Journalist usw.), in der Regel fehlen. Berufschancen bieten jedoch vorwiegend die kleinen bzw. mittelständischen deutschen Unternehmen, in denen im wesentlichen Geschäfte zwischen China und Deutschland abgewickelt werden. Denn die Abhängigkeit der kleinen bzw. mittelständischen Unternehmen von ihrem deutschen Mutterhaus hat zur Folge, daß sie deutschsprechendes Ortspersonal bevorzugen. Demgegenüber ist in den multinationalen deutschen Unternehmen Englisch die lingua franca. Immerhin sind Kenntnisse der deutschen Sprache ein Plus bei der Bewerbung und können bei der Arbeit eine bessere Verständigung mit der deutschen Führungsspitze schaffen. Englischkenntnisse sind aber auf jeden Fall wichtig, und dies müßte bei der Entwicklung der Curricula in Betracht gezogen werden. Infolge der besseren deutschen Sprachund Kulturkenntnisse aus der Ausbildung werden GermanistInnen vor allem in den Verwaltungsabteilungen einge- 
setzt. Die mit der Büroarbeit häufig verbundenen mündlichen Kompetenzen wie z.B. Telefonateführen, Besprechungen und Gespräche zur Kontaktpflege reichen jedoch auf internationalem $\mathrm{Ni}$ veau nicht aus. Folglich werden praxisnahe Interaktionsübungen und berufsbezogene mündliche Trainings nachdrücklich für den Deutschunterricht vorgeschlagen.

Briefe, z. B. Einladungen oder Danksagungen, Geschäftsbriefe, Berichte, Protokolle/Memoranden und Anweisungstexte sind die häufigsten geschriebenen Textsorten im Geschäftsleben. Das selbständige Schreiben der chinesischen MitarbeiterInnen wird zwar häufig verlangt, ein anschließendes Überarbeiten durch deutsche MuttersprachlerInnen ist jedoch kein seltener Fall. Die Probleme konzentrieren sich in erster Linie auf die Stilebene, nämlich die Texte textsortenentsprechend und situationsangemessen $\mathrm{zu}$ verfassen. Insbesondere Geschäftsbriefe, Protokolle/Memoranden und Berichte sollen ins Zentrum des Schreibunterrichts gerückt werden.

Erwartungsgemäß werden mehr Texte gelesen als geschrieben. Beim Lesen sind Briefe zur Kontaktpflege, Geschäftsbriefe und Anweisungstexte ebenso wichtig wie beim Schreiben, darüber hinaus fallen Pressemitteilungen und Produkt-/ Betriebsbroschüren ins Gewicht. Zu den wichtigsten Inhalten im Unterricht gehören Geschäftsbriefe und Protokolle/Memoranden, wobei die StudentInnen auch für das Verstehen der Struktur und Form eines Vertrags sensibilisiert werden sollen. Auf Berichte, jounalistische Wirtschaftstexte und Artikel in Fachzeitschriften soll ebenfalls Rücksicht genommen werden, obwohl sie nicht zu den am häufigsten gelesenen Texten zählen.

Daß ein Viertel der GermanistInnen in der Wirtschaft in der Handelsabteilung ihren Platz einnehmen, signalisiert den weiteren Spielraum in den Unternehmen für die GermanistikstudentInnen. Dies erfordert aber unbedingt Kenntnisse des wirtschaftlichen know-hows. Auch Dolmetsch- und Übersetzungsaufgaben, die bei der Hälfte der Unternehmen anfallen, bedürfen wirtschaftlicher Fachlexik und Fachkenntnisse in allen möglichen Wirtschaftsbereichen. Jedoch ist eine tiefgreifende Ausbildung in Wirtschaftswissenschaften nicht gefordert. Es wird gewünscht, daß die GermanistikstudentInnen wirtschaftliche Grundkenntnisse erwerben und so Voraussetzungen für die spätere berufliche Weiterbildung besitzen. Betriebswirtschaftliches Wissen ist für ein konkretes Unternehmen wichtiger als volkswirtschaftliche Kenntnisse, die man nach der Aneignung der Grundbegriffe auch durch die Medien erweitern kann.

Im Unterricht für Wirtschaftsdeutsch soll außerdem die interkulturelle Komponente eingebunden werden. Chinesische MitarbeiterInnen, die Kenntnisse über das Wirtschaftssystem, die Wirtschaftsstruktur sowie die Wirtschaftslandeskunde in China mitbringen, werden in den deutschen Unternehmen sehr begrüßt. Der Handlungsfähigkeit und der selbständigen Arbeitskompetenz, die in der Wirtschaft oft sehr hoch bewertet und bei den chinesischen MitarbeiterInnen bisher als mangelhaft erlebt werden, soll in der Ausbildung genügend Rechnung getragen werden.

Ich gehe davon aus, daß die Ergebnisse dieser Befragung, die noch durch weitere aus anderen Regionen Chinas ergänzt werden sollen, eine tragfähige Basis liefern können zur Entwicklung eines Curriculums für diejenigen Universitäten in China, die eine Germanistik-Ausbildung anbieten wollen, die auf den Arbeitsmarkt in der Wirtschaft abgestimmt ist. 


\section{Literatur}

Atteslander, Peter: Methoden der empirischen Sozialforschung. Berlin; New York: de Gruyter, 1984.

Bea, Franz Xaver; Dichtl, Erwin; Schweitzer, Marcell (Hrsg.): Allgemeine Betriebswirtschaftslehre. - Bd. 1: Grundfragen. Stuttgart: Lucius \& Lucius, 1997 (Uni-Taschenbücher 1081).
Forschungsprojekt »Deutsch-finnische Kulturunterschiede in der Wirtschaftskommunikation «: Zwischenbericht. Helsinki: Helsingin Kauppakorkeakulun julkaisuja, 1989.

Zhao, Jin: Wirtschaftsdeutsch im Germanistikstudium - Linguistische Analyse und didaktische Überlegungen. Magisterarbeit. Tongji-Universität Schanghai, 1996.

\section{Fragebogen}

\section{Hintergrundinformationen zu den Befragten}

1. Was ist Ihre jetzige Tätigkeitsbezeichnung?

2. Welche der folgenden Ebenen entspricht am besten Ihrer Stellung?
1. Geschäftsführung/Vorstand
2. (Haupt)abteilungsleitung
3. (Unter)abteilungsleitung
4. SachbearbeiterIn
5. SekretärIn
6. Sonstige, welche?

3. Ist Ihre Muttersprache
a) Chinesisch?
b) Deutsch?
c) Englisch?

\section{Hintergrundinformationen zur Firma}

4a. Wirtschaftssektoren
1. Industrie
welche?
2. Handel

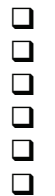
3. Transport
4. Bau
5. Dienstleistungen
welche?
6. Banken
7. Sonstige, welche?

$\square$
$\square$
$\square$
$\square$
$\square$

4b. Unternehmensform Ihres Unternehmens
1. Equity Joint Ventures
2. Contractual Joint Ventures
3. Wholly Foreign-Owned Enterprises
4. Representative Office

5a. Anzahl der Mitarbeiter, davon chinesische Mitarbeiter()
口 0-4 ( )
$\square$ 5-9 ( )
$\square$ 100-199 ( )
$\square$ 10-19( )
$\square$ 200-499 ( )
$\square 20-49(\quad)$
$\square 500$ bis ( )

$5 b$. Hintergrund der chinesischen Mitarbeiter
1. Fachpersonal ohne Deutschkenntnisse
2. Fachpersonal mit Deutschkenntnissen
3. Deutschstudenten (Germanisten)

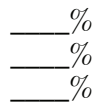


5c. In welcher Abteilung arbeiten die meisten Deutschstudenten?
1. Produktionsabteilung
2. Handelsabteilung
3. Verwaltungsabteilung

6. Bedeutung der Geschäftsbeziehungen mit Deutschland, Anteil am Umsatz:
a) unter $25 \%$
b) $25-49 \%$
c) $50-75 \%$
d) $76-100 \%$

\section{Sprachverwendung}

7a. Wie oft verwenden die chinesischen Mitarbeiter in der mündlichen Kommunikation mit ihren deutschen Geschäftspartnern folgende Sprachen:

$$
4=\text { fast immer, } 3=\text { recht viel, } 2=\text { etwas, } 1=\text { nie }
$$

Deutsch

Englisch

Chinesisch

$$
\begin{array}{llll}
4 & 3 & 2 & 1
\end{array}
$$

7b. Haben die chinesischen Mitarbeiter in der mündlichen Kommunikation in Deutsch
4. keine Schwierigkeiten
3. Schwierigkeiten
2. geringe Schwierigkeiten
1. große Schwierigkeiten

8. Welche Sprache verwenden die chinesischen Mitarbeiter in erster Linie in den folgenden Kommunikationssituationen mit ihren deutschen Geschäftspartnern.

Verhandlungen
Deutsch
Englisch
Chinesisch

Besprechungen

Telefonate

Unternehmenspräsentationen

Produktpräsentationen

Informelle Zusammenkünfte

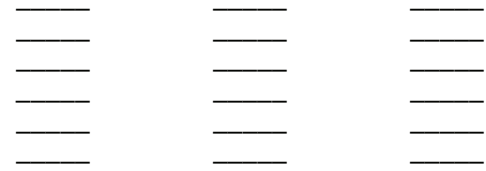

9. Welche Sprachen werden gedolmetscht:

Englisch-Chinesisch

und welchen Anteil hat das Dolmetschen in Ihrer Firma:
4. sehr groß
3. groß
2. mittelgroß $\square$
1. gering

10. Wie oft verwenden die chinesischen Mitarbeiter im deutschen Handel in der schriftlichen Kommunikation die folgenden Sprachen.

4 = fast immer, $3=$ recht viel, $2=$ selten, $1=$ nie

Deutsch

$$
\begin{array}{llll}
4 & 3 & 2 & 1
\end{array}
$$

Englisch

Chinesisch

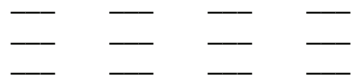

11. Welche schriftlichen deutschsprachigen Texte schreiben und lesen die chinesischen Mitarbeiter? Geben Sie bitte die Häufigkeit jeweils getrennt folgendermaßen an:

4 = wöchentlich, 3 = monatlich, 2 = jährlich, 1 = nie

\section{A. Geschriebene Texte}

Geschäftsbriefe

Sonstige Briefe (Einladungen,

Danksagungen u. a.)

Berichte verschiedener Art

Protokolle u. Memoranden

Anweisungstexte
B. Gelesene Texte

Geschäftsbriefe

Sonstige Briefe (Einladungen,

Danksagungen u. a.)

Berichte verschiedener Art

Protokolle u. Memoranden

Anweisungstexte 
Verträge

Produkt- und Betriebsbroschüren

Pressemitteilungen

Journalistische Wirtschaftstexte

Artikel in Fachzeitschriften

Sonstige, welche?
Verträge

Produkt- und Betriebsbroschüren

Pressemitteilungen

Journalistische Wirtschaftstexte

Artikel in Fachzeitschriften

Sonstige, welche?

12. Wie ist der übliche Arbeitsablauf, wenn die chinesischen Mitarbeiter deutschsprachige Texte schreiben? Geben Sie bitte die drei häufigsten Vorgehensweisen entsprechend an: 1 = am häufigsten, 2 = am zweithäufigsten, 3 = am dritthäufigsten

1. Sie schreiben den Text von Anfang bis Ende selbst.

2. Sie schreiben den Text oder einen Entwurf auf Chinesisch und jemand anders fertigt eine deutschsprachige Fassung an.

3. Sie erstellen einen Entwurf in Deutsch und jemand anders überarbeitet ihn.

4. Sie nehmen einen früher erstellten Text als Muster.

5. Sonstige, welche?

13. Empfinden die chinesischen Mitarbeiter das Schreiben deutschsprachiger Texte als schwierig?
a.) ja
b.) nein
b.) Wenn ja, was bereitet beim Schreiben besondere Schwierigkeiten?
1. Grammatik
2. Wortschatz
3. Fachlexik
4. Stil $\square$
c.) Gibt es Unterschiede beim Schreiben von Briefen, Telefax oder e-mail?

14. Welche Sprachen werden übersetzt:
Englisch-Chinesisch
Deutsch-Chinesisch

Wie groß ist der Anteil des Übersetzens in Ihrer Firma?
4. sehr groß
3. groß
2. mittelgroß $\square$
1. gering $\square$

15. Wie ausführlich sollten die unten angegebenen Texttypen im deutschen Sprachunterricht der Hochschulen behandelt werden? Geben Sie bitte nach Wichtigkeit an

$5=$ sollte vorrangig im Unterricht behandelt werden

$4=$ ein beträchtlicher Teil des Unterrichts sollte sich damit befassen

3 = wenigstens ein kleiner Teil des Unterrichts sollte dafür verwendet werden

$2=$ wenn zeitlich möglich, könnten diese Texte etwas behandelt werden

$1=$ lohnt sich nicht, diese Texte im Unterricht zu behandeln

A. Geschriebene Texte

Geschäftsbriefe

Sonstige Briefe (Einladungen,

Danksagungen u. a.)

Berichte verschiedener Art

Protokolle $\mathrm{u}$. Memoranden

Anweisungstexte

Verträge

Produkt- und Betriebsbroschüren

Pressemitteilungen

Journalistische Wirtschaftstexte

Artikel in Fachzeitschriften

Sonstige, welche?
B. Gelesene Texte

Geschäftsbriefe

Sonstige Briefe (Einladungen,

Danksagungen u. a.)

Berichte verschiedener Art

Protokolle u. Memoranden

Anweisungstexte

Verträge

Produkt- und Betriebsbroschüren

Pressemitteilungen

Journalistische Wirtschaftstexte

Artikel in Fachzeitschriften

Sonstige, welche?

16. Welche wirtschaftlichen Fachkenntnisse sollten Ihrer Meinung nach die chinesischen Mitarbeiter haben? Fügen Sie bitte ein:

5 = unverzichtbar, $4=$ sehr wichtig, 3 = wichtig, 2 = weniger wichtig, 1 = überflüssig

Betriebswirtschaftslehre:

a) Grundbegriffe (betriebliche Produktionsfaktoren, Funktionsbereiche wie

Produktion, Beschaffung, Absatz etc.) 
b) Industriebetriebslehre (Materialwirtschaft, Produktion, Absatzwirtschaft, Investition und Finanzierung etc.)

c) Handelsbetriebslehre (insbesonders BWL des Außenhandelsbetriebes)

d) Bankbetriebslehre

e) Personalwirtschaft

f) Finanzbuchhaltung

g) Kalkulation und Kostenrechnung

h) Transportwesen

i) Betriebliche Steuerlehre

j) Sonstiges

Volkswirtschaftslehre

a) Grundbegriffe (Bedürfnisse, Güter, Produktion, Konsum, Knappheit, Wirtschaftssubjekte etc.)

b) Wirtschaftskreislauf

c) Volkswirtschaftliche Gesamtrechnung (Volkseinkommen, Bruttosozialprodukt etc.)

d)Markt und Preis

e) Geld, Kredit, Banksystem

f) Außenwirtschaftslehre und -politik

g) Konjunktur und Wachstum

h) Wirtschaftssysteme

i) Steuern in Deutschland und China

j) Wirtschaftspolitik

k) Wirtschaftsentwicklung und -struktur in Deutschland und China

1) Sonstiges

17. Was sollte Ihrer Meinung nach im Deutschunterricht an der Hochschule behandelt werden, um die Deutschstudenten am besten für die kommenden Aufgaben im Geschäftsleben vorzubereiten?

a) Schriftliche Kommunikation

b) Mündliche Kommunikation

c) Kultur der deutschsprachigen Länder (Vergleich zur Kultur Chinas)

d) Wirtschaftliche Fachkenntnisse

e) Sonstiges 